\title{
BumNerdoby oenen Comparison of neurofilament light chain results between two independent facilities
}

\author{
Tobias Sejbaek (10 , ${ }^{1,2}$ Jason P Mendoza, ${ }^{3}$ Natasha Penner, ${ }^{3}$ Jonna Skov Madsen, ${ }^{4,5}$ \\ Dorte Aalund Olsen, ${ }^{5}$ Zsolt Illes (i) ${ }^{6}$
}

To cite: Sejbaek T, Mendoza JP, Penner N, et al. Comparison of neurofilament light chain results between two independent facilities. BMJ Neurology Open 2020;2:e000063. doi:10.1136/ bmjno-2020-000063

Accepted 23 September 2020

Check for updates

(C) Author(s) (or their employer(s)) 2020. Re-use permitted under CC BY-NC. No commercial re-use. See rights and permissions. Published by BMJ.

${ }^{1}$ Neurology, Hospital South West Jutland, University Hospital of Southern Denmark, Esbjerg, Denmark

${ }^{2}$ BRIDGE, Department of Regional Health Research, University of Southern Denmark, Odense, Denmark

${ }^{3}$ Biogen, Cambridge

Massachusetts, USA

${ }^{4}$ Department of Regional Health Research, University of Southern Denmark, Odense, Denmark

${ }^{5}$ Department of Biochemistry and Immunology, Lillebælt Hospital, University Hospital of Southern Denmark, Vejle, Denmark

${ }^{6}$ Department of Neurology, University of Southern Denmark, Odense, Denmark

Correspondence to Dr Tobias Sejbaek;

Tobias.Sejbaek.Mathiesen@ rsyd.dk

\section{ABSTRACT}

Objectives To examine levels of neurofilament light chain (NFL) in identical cerebrospinal fluid (CSF) and blood samples at two different facilities, and how differences affect interpretation of levels within and above the normal range.

Methods CSF and plasma from patients with multiple sclerosis (MS) and healthy controls (HCs) were analysed by Simoa (Quanterix) for levels of NFL providing a total of 165 CSF samples (119 from MS) and 225 plasma samples (180 from MS).

Results CSF and plasma concentrations highly correlated between NFL laboratory facilities (R: 0.92 and 0.84 , $<0.0001$, respectively), and there were no differences between facilities. A bias between the two sites for plasma was $-0.95 \mathrm{pg} / \mathrm{mL}$ and for CSF $-73.53 \mathrm{pg} / \mathrm{mL}$. The cutoffs for CSF were 807.5 and $571.0 \mathrm{pg} / \mathrm{mL}$ at site 1 and site 2, respectively; the cut-offs for plasma were 13.0 and $11.8 \mathrm{pg} / \mathrm{mL}$, respectively. Seven out of 180 plasma samples (3.9\%) and 3 out of 119 CSF samples (2.5\%) from MS patients could be reclassified as normal/abnormal, that is, below/above cut-off, when measured at different facilities.

Conclusion Our study demonstrates that results of NFL in CSF and blood measured with SIMOA are comparable between facilities. Nevertheless, healthcare practitioners should consider reference values at different laboratories, since different sensitivity/specificity can affect interpretation when low values are adjacent to cut-offs.

\section{INTRODUCTION}

Neurofilament light chain (NFL) is a nonspecific marker for neuroaxonal damage that is currently being investigated in several neurological diseases as a possible tool for disease monitoring and management. ${ }^{1-4}$ NFL levels measured by different technologies have been validated, and the most sensitive commercially available assay is the single molecule array (SIMOA) from Quanterix (Massachusetts, USA). ${ }^{56}$ We examined differences between NFL levels in 165 cerebrospinal fluid (CSF) and 225 blood samples measured with the commercially available assay SIMOA at two different laboratories; we addressed if potential differences affect the interpretation of presumed clinical activity in multiple sclerosis (MS) based on NFL levels.

\section{METHODS}

Study design

The Tecfidera in Relapsing-Remitting Multiple Sclerosis: Endothelial Dysfunction (TREMEND) study is a prospective openlabel phase IV trial, which enrolled newly diagnosed patients with MS with relapsingremitting multiple sclerosis from March 2014 until August 2016 (EudraCT 2014-000254-11) and treated with dimethyl fumarate (DMF) for 24 months. ${ }^{7}$

\section{Sample collection NFL analysis}

Plasma were collected at baseline, 3, 6, 12 and 24 months of treatment with DMF from patients enrolled in the TREMEND trial $(n=52)$ and from healthy controls (HCs) $(n=23)$. CSF was collected, however optional as part of the TREMEND trial at baseline and after 12 months of DMF treatment. Samples from baseline and 12 months were analysed for NFL at two different facilities. Patients were $34.1( \pm 8.7)$ years old and $86.6 \%$ women; HCs were on average $38.2( \pm 11.2)$ years old and $89.2 \%$ women.

Samples were collected following international guidelines for biobanking; venous blood was drawn in evacuated K2-EDTA containing blood collection tubes and both blood and CSF were centrifuged within 30-60 min after collection at 2000 $\mathrm{G}$ for $10 \mathrm{~min}$ at $20^{\circ} \mathrm{C}$, plasma and CSF were aliquoted in $500 \mu \mathrm{L}$ Sarstedt polypropylene tubes and stored at $-80^{\circ} \mathrm{C}$ until batch analysis. ${ }^{8}$ Blood and CSF samples were taken from the cubital vein and by spinal tap.

\section{NFL analysis}

NFL was analysed in the same batch at each facility. ${ }^{9}$ Samples were thawed once before 
aliquoting and shipping, and once again before analysing at the two laboratory facilities.

A commercially available NFL kit (Quanterix, Lexington, Massachusetts, USA) for the Single Molecule Array (Simoa) HD-1 Analyzer (Quanterix) was used to quantify NFL light chain in serum and CSF according to the manufacturer's procedure. The results from site 1 (Quanterix) have previously been published. ${ }^{7}$ Samples were reanalysed at the Department of Biochemistry and Immunology, Lillebaelt Hospital, Vejle, a routine laboratory being accredited by Danish Accreditation Fund (DANAK) according to the ISO 15189 standard. Quality control was performed using two controls prepared from commercially available control material provided by the manufacturer. In-house serum and CSF pools were used as internal controls (plasma pool $25 \mathrm{pg} / \mathrm{mL}$ and CSF pool $1350 \mathrm{pg} / \mathrm{mL}$ ) and included in each assay for evaluating assay performance. Samples analysed at site 1 (Quanterix) were analysed in single measures while at site 2 in duplicates and the mean was calculated. The intermediate precision was $<9.3 \%$ at site 1 and $<12 \%$ at site 2 . Lower limit of detection was $0.038 \mathrm{pg} / \mathrm{mL}$ and lower limit of quantification was $0.174 \mathrm{pg} / \mathrm{mL}$ at both laboratories. The intermediate precision expresses within-laboratory variations: different days, different analysts, different reagent lots, and so on.

\section{Standard protocol approvals, registrations, patient consents and monitoring}

All patients and HCs gave written and oral consent. The trial was monitored according to the national laws by the unit for good clinical practice at Odense University
Hospital, Odense, Denmark, and South West Jutland University Hospital, Esbjerg, Denmark.

\section{Statistical analyses}

We described baseline characteristics with means and SD for continuous variables. Linear fit regression was performed using Spearman linear fit regression to calculate coefficients and linearity between NFL in CSF and plasma. Data were checked for normality using D'Agostino and Pearson normality test. Bland-Altman analysis was performed to compare differences between the two laboratory sites and evaluate bias. Receiver operating characteristic (ROC) analysis was performed to identify differences in cut-offs, specificity and sensitivity between the two NFL laboratory facilities. We performed nonparametric analysis with Kruskal-Wallis test and Dunn's multiple comparisons. Statistical analysis and data management were performed using GraphPad Prism V.8.

\section{RESULTS}

NFL concentrations were analysed and compared in 165 CSF and 225 plasma samples measured by SIMOA method at two different laboratory facilities (sites), respectively (table 1; figure 1A,B). Baseline NFL levels in patients with MS were $2368( \pm 1947) \mathrm{pg} / \mathrm{mL}$ and $16.4( \pm 14.4)$ $\mathrm{pg} / \mathrm{mL}$ in CSF and plasma, respectively, compared with lower levels in HCs $(416( \pm 191)$ and $7.3( \pm 3.0)$ in CSF and plasma, respectively) $(\mathrm{p}<0.0001$ in both CSF and plasma). Linear regression was used for correlation, and agreement between the two assays at the two sites was examined by Bland-Altman analysis (figure $1 \mathrm{C}-\mathrm{F}$ ). We found

Table 1 Comparison of NFL levels in plasma and CSF measured at two different NFL laboratory facilities

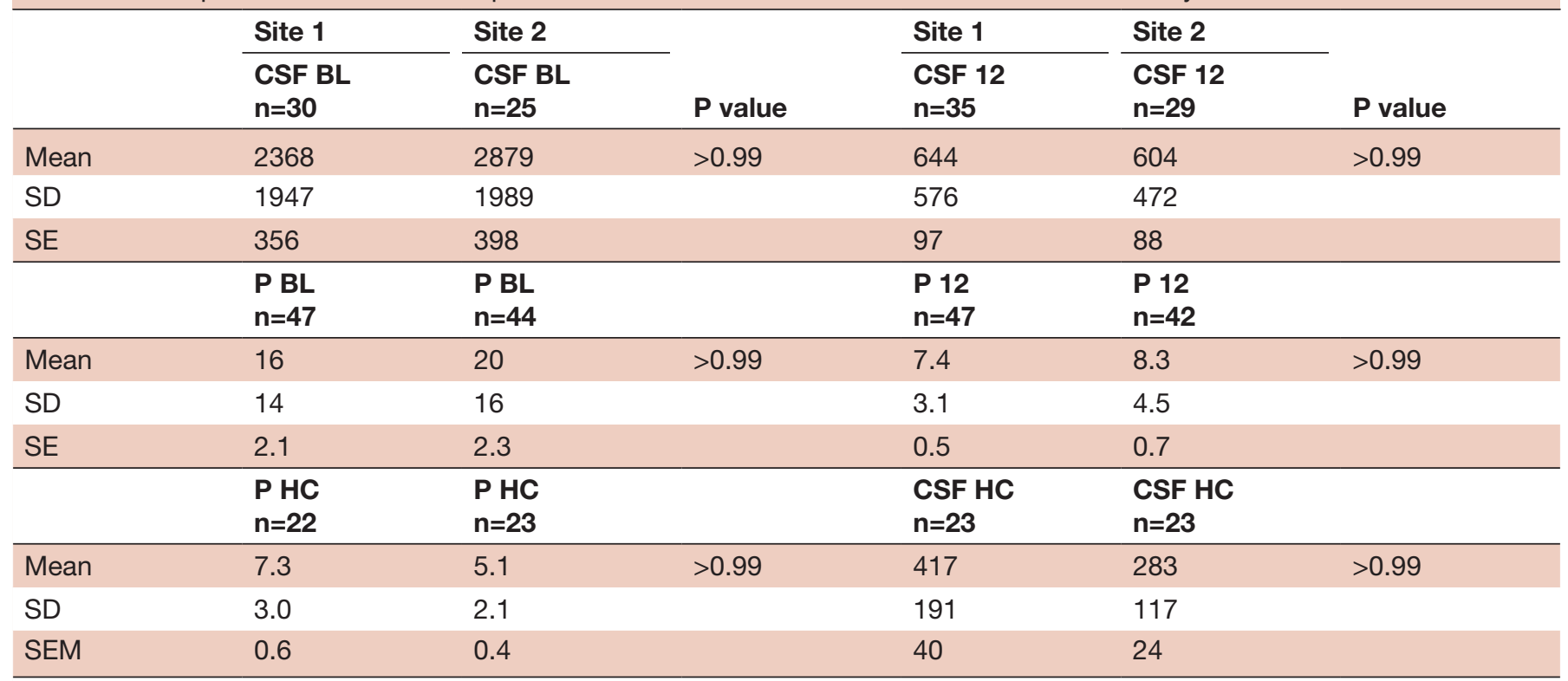

Dunn's multiple comparisons test comparing the levels of NFL (pg/mL) in CSF (total number 165) and plasma (total number 225), between two sites. Samples were from patients with multiple sclerosis, before and after 12 months of treatment with dimethyl fumarate and a HC group. Site 1 and site 2 are two independent NFL laboratories. P12 and CSF12=samples at 12 months.

$\mathrm{BL}$, baseline samples; CSF, cerebrospinal fluid; HC, healthy control; n, number; NFL, neurofilament light chain; P, plasma. 

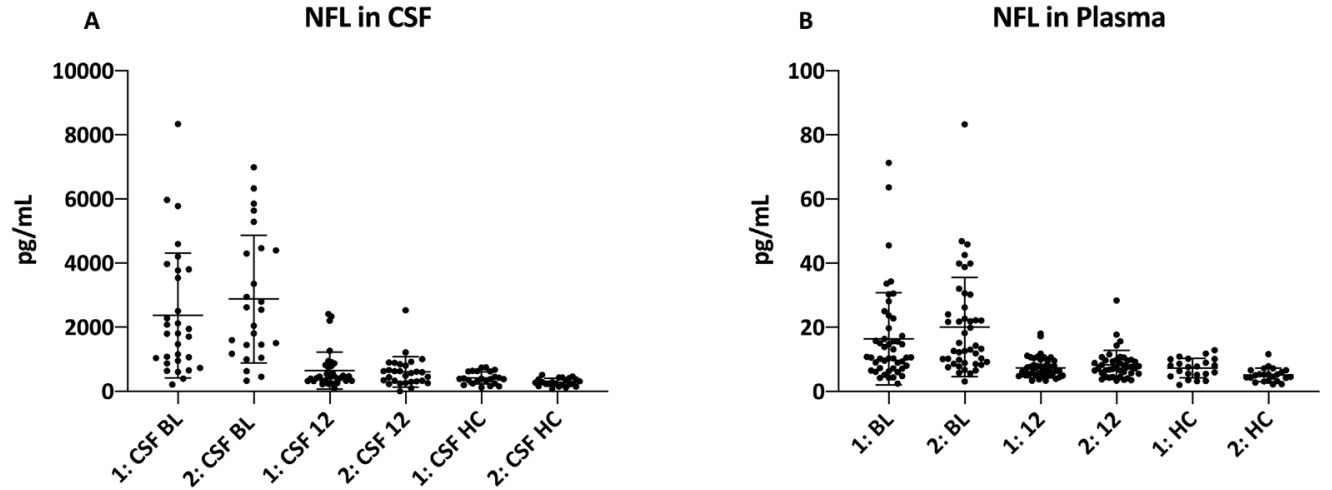

\section{Correlation of NFL in CSF}
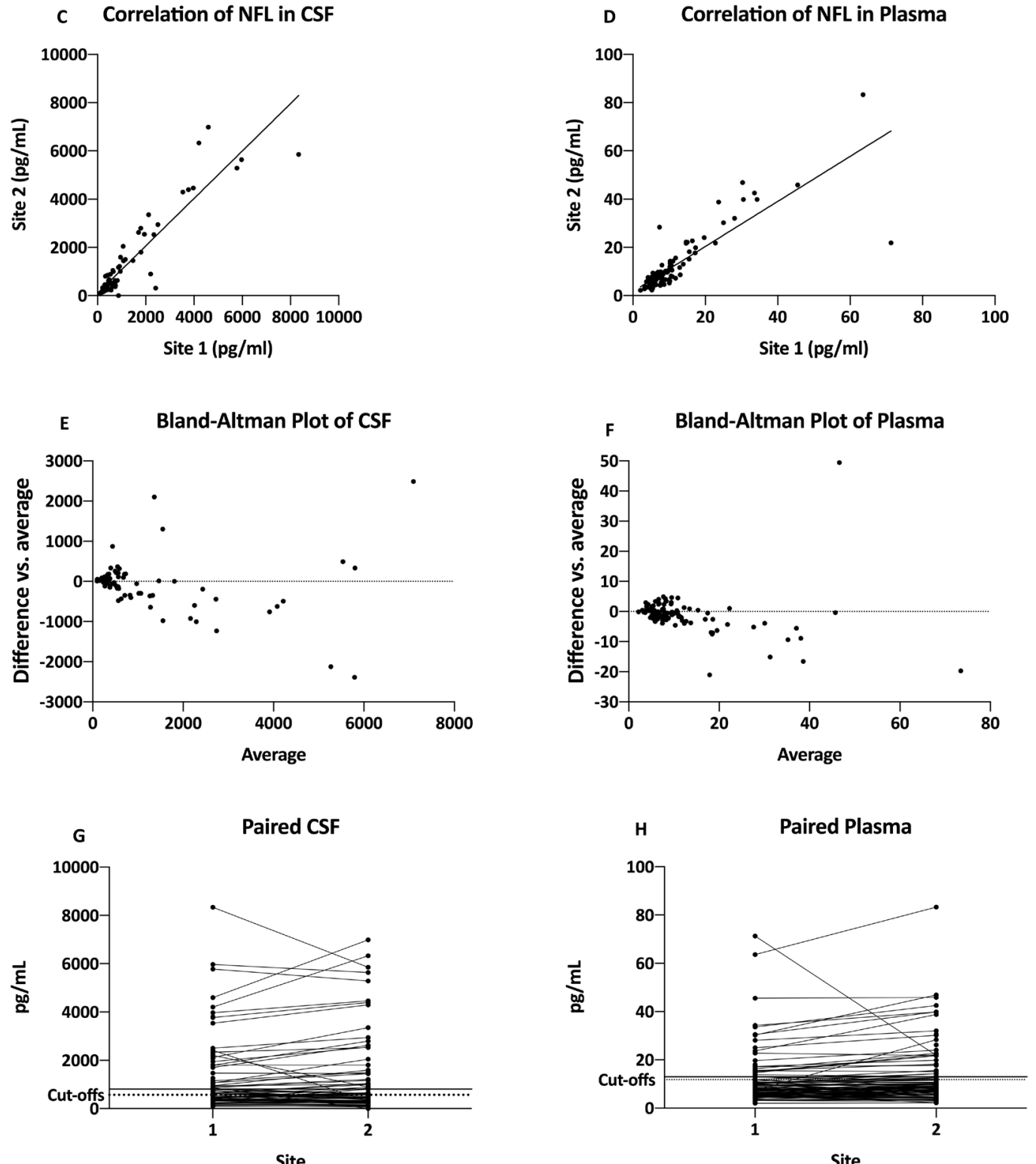

Site

Site

Figure 1 Comparison of neurofilament light chain (NFL) levels in cerebrospinal fluid (CSF) and plasma measured at two different facilities. (A and B) Scatter dot plot with levels of NFL at baseline (BL), after 12 months (12) treatment with dimethyl fumarate (DMF), and in healthy controls $(\mathrm{HC})$ at site 1 and site 2. Whiskers indicate SD and mean. (C and D) The figure depicts linear regression analysis of paired samples at site 1 and site 2. ( $E$ and F) Bland-Altman plot with differences between sites (site 1 minus site 2) versus average are shown. ( $F$ and G) Paired NFL measured in the same samples at site 1 and site 2 are shown. In three samples, CSF NFL were below cut-off $(807.5 \mathrm{pg} / \mathrm{mL})$ at site 1 and above cut-off $(571.0 \mathrm{pg} / \mathrm{mL})$ at site 2. NFL levels in six plasma samples were below cut-off $(13.0 \mathrm{pg} / \mathrm{mL})$ at site 1 and at above cut-off $(11.8 \mathrm{pg} / \mathrm{mL})$ at site 2 . NFL level in a single plasma sample was above cut-off at site 1 and below cut-off at site 2 . 
that both CSF and plasma concentrations were highly correlated between NFL laboratory facilities (R: 0.92 and $0.84, \mathrm{p}<0.0001$, respectively), and we did not find significant differences between the two sites (figure 1C-D). A systematic bias between the two sites calculated by the Bland-Altman analysis was $-0.95 \mathrm{pg} / \mathrm{mL}$ for plasma and $-73.53 \mathrm{pg} / \mathrm{mL}$ for CSF. Site 2 measured lower concentration of NFL in both plasma and CSF compared with site 1. When NFL concentration in identical CSF and plasma samples measured at the two sites were compared, only minor differences were found apart from a few outliers (1\%-2\% of samples) (figure $1 \mathrm{G}, \mathrm{H})$.

Finally, we examined if interpretation of results may change, when the same samples are examined for NFL at the different facilities with their own calculated cut-offs, specificity and sensitivity based on the results (figure 1G,H). We therefore calculated cut-off values at both laboratories by ROC analysis for both plasma and $\mathrm{CSF}^{7}$ The cut-offs for CSF were 807.5 and $571.0 \mathrm{pg} / \mathrm{mL}$ at site 1 and site 2, respectively; the cut-offs for plasma were 13.0 and $11.8 \mathrm{pg} / \mathrm{mL}$, respectively. For CSF, we found a sensitivity of $80.0 \%$ (95\% CI: $62.7 \%$ to $90.5 \%$ ) and a specificity of $100.0 \%$ (95\% CI: $85.7 \%$ to $100.0 \%$ ) at site 1 , whereas site 2 demonstrated a higher sensitivity of $92.0 \%$ (95\% CI: $75.0 \%$ to $98.6 \%$ ) with the same specificity of $100.0 \%$ (95\% CI: $85.7 \%$ to $100.0 \%$ ). For plasma, we found a sensitivity of $46.8 \%$ (95\% CI: $33.3 \%$ to $60.8 \%$ ) and a specificity of $100.0 \%$ (95\% CI: $85.1 \%$ to $100.0 \%$ ) at site 1 , whereas site 2 demonstrated again higher sensitivity of $63.6 \%$ (95\% CI: $48.9 \%$ to $76.2 \%$ ) with the same specificity of $100.0 \%$ (95\% CI: $85.7 \%$ to $100.0 \%$ ). Area under ROC curve for CSF was 0.93 (SE: 0.035, $\mathrm{p}<0.0001$ ) and 0.98 (SE: 0.016, $\mathrm{p}<0.0001$ ) for sites 1 and 2, respectively. Area under ROC curve for plasma was 0.76 (SE: $0.057, \mathrm{p}<0.001)$ and 0.94 (SE: $0.027, \mathrm{p}<0.0001$ ) for sites 1 and 2 , respectively.

The concentrations of NFL in individual samples were then examined by using these established cut-off values at both sites. We found that 7 out 180 plasma samples $(3.9 \%)$ could be reclassified as normal/abnormal, that is, below/above cut-off, when measured at different sites (figure 1H). Similarly, 3 out of 119 CSF samples (2.8\%) could be reclassified as normal/abnormal (figure 1G).

\section{DISCUSSION}

We determined NFL levels in identical CSF and plasma samples at two independent laboratory facilities using the commercially available highly sensitive assay from Quanterix. No differences between these laboratory facilities could be found on the group level (table 1). Linear fit regression demonstrated good correlation between facilities, however with better correlation in CSF than in plasma. We found a small bias (for plasma $-0.95 \mathrm{pg} / \mathrm{mL}$ and for CSF $-73.53 \mathrm{pg} / \mathrm{mL}$ ) with one laboratory facility determining lower concentrations in both plasma and CSF than the other site. This bias was also reflected in the difference between the identified cut-offs. Bias between laboratory facilities is well documented for NFL and other biomarkers even with higher variation than in our study. ${ }^{10}$ Site 2 had a slightly higher sensitivity in both plasma and CSF compared with site 1 . Such differences in cut-offs and sensitivity/specificity resulted in a reclassification of approximately $3 \%-4 \%$ of patients' samples below and above cut-off in plasma and CSF, while none of the HC samples were reclassified. We have recently shown that $22 \%$ of patients with normal NFL levels in the plasma had elevated levels in the paired CSF sample. ${ }^{7}$ Our present data add another factor to the complexity of interpreting NFL levels in patients with MS. These data altogether indicate that in a small percentage of patients with MS, variability in laboratory methods and lower sensitivity in the plasma may result in interpretation of 'no clinical activity' based on plasma NFL, while CSF or a different laboratory may conclude the opposite. Therefore, standardisation of methodology, individual cut-offs or percentiles at different laboratory facilities, and regular sampling of blood for NFL may be required. ${ }^{11} 12$

This is, to our knowledge, the first study comparing NFL levels in identical paired samples from CSF and plasma at two NFL laboratory facilities. Our results demonstrate that healthcare practitioners should take into account the local reference values, when comparing consecutive levels of NFL in their clinical practice. However, differences tend to be minor between facilities, and only low values around cut-offs should be interpreted with caution.

This study is not without limitations. Sera was not analyzed, although it might provide better option than plasma: NFL concentration is $23 \%$ higher in sera, and the clinical implication might be different in sera compared to plasma.

In conclusion, our study demonstrates that results of NFL in CSF and blood measured with SIMOA from Quanterix are comparable between two different NFL laboratory facilities. Nevertheless, healthcare practitioners should consider reference values from different laboratory facilities especially, when consecutive samples analysed by different facilities are compared. Different sensitivity/specificity can affect data interpretation in $4 \%$ of cases, when results are around cut-offs, however on an individual patient level, effort should be made to use the same NFL laboratory facility. These data warrant additional larger studies comparing different variables reflective of clinical practice.

Collaborators Multiple Sclerosis Alliance of Southern Denmark: Stenager E, Illes Z, Jensen HB, Kant M, Petersen A.

Funding This trial was funded by research grants from Biogen (TREMEND to ZI and TS), University of Southern Denmark (14/24200 to TS and ZI), Odense University Hospital (5798002573633 to TS and ZI), The Danish Multiple Sclerosis Society (R367-A25341 to ZI), OUH/Rigshopitalet grant (to ZI), Regions of Southern Denmark, Clinical Researcher 2018 (to TS).

Competing interests TS has served on scientific advisory boards, received support for congress participation, received speaker honoraria and received research support from Biogen and Novartis. NP is an employee of and holds stock/ stock options in Biogen. JPM is an employee of and holds stock/stock options in Biogen. Zl has served on scientific advisory boards, served as a consultant, 
received support for congress participation, received speaker honoraria and received research support from Biogen, Merck-Serono, Sanofi-Genzyme, Lundbeck and Novartis.

Patient consent for publication Not required.

Ethics approval This study was approved by the Danish Data Protection Agency (journal no 17/12684) and the Southern Danish Committee on Health Research Ethics (S-20140015).

Provenance and peer review Not commissioned; externally peer reviewed.

Data availability statement Data are available upon reasonable request.

Open access This is an open access article distributed in accordance with the Creative Commons Attribution Non Commercial (CC BY-NC 4.0) license, which permits others to distribute, remix, adapt, build upon this work non-commercially, and license their derivative works on different terms, provided the original work is properly cited, appropriate credit is given, any changes made indicated, and the use is non-commercial. See: http://creativecommons.org/licenses/by-nc/4.0/.

\section{ORCID iDs}

Tobias Sejbaek http://orcid.org/0000-0002-7682-2188

Zsolt Illes http://orcid.org/0000-0001-9655-0450

\section{REFERENCES}

1 Khalil M, Teunissen CE, Otto M, et al. Neurofilaments as biomarkers in neurological disorders. Nat Rev Neurol 2018;14:577-89.

2 Lycke JN, Karlsson JE, Andersen O, et al. Neurofilament protein in cerebrospinal fluid: a potential marker of activity in multiple sclerosis. J Neurol Neurosurg Psychiatry 1998;64:402-4.
3 Mattsson N, Andreasson U, Zetterberg H, et al. Association of plasma neurofilament light with neurodegeneration in patients with Alzheimer disease. JAMA Neurol 2017;74:557-66.

4 Weydt P, Oeckl P, Huss A, et al. Neurofilament levels as biomarkers in asymptomatic and symptomatic familial amyotrophic lateral sclerosis. Ann Neurol 2016;79:152-8.

5 Disanto G, Barro C, Benkert P, et al. Serum neurofilament light: a biomarker of neuronal damage in multiple sclerosis. Ann Neurol 2017;81:857-70.

6 Kuhle J, Barro C, Andreasson U, et al. Comparison of three analytical platforms for quantification of the neurofilament light chain in blood samples: ELISA, electrochemiluminescence immunoassay and Simoa. Clin Chem Lab Med 2016;54:1655-61.

7 Sejbaek T, Nielsen HH, Penner N, et al. Dimethyl fumarate decreases neurofilament light chain in CSF and blood of treatment naïve relapsing MS patients. J Neurol Neurosurg Psychiatry 2019;90:1324-30.

8 Teunissen CE, Petzold A, Bennett JL, et al. A consensus protocol for the standardization of cerebrospinal fluid collection and biobanking. Neurology 2009;73:1914-22.

9 Gisslén M, Price RW, Andreasson U, et al. Plasma concentration of the neurofilament light protein (NFL) is a biomarker of CNS injury in HIV infection: a cross-sectional study. EBioMedicine 2016;3:135-40.

10 Waters P, Reindl M, Saiz A, et al. Multicentre comparison of a diagnostic assay: aquaporin-4 antibodies in neuromyelitis optica. $J$ Neurol Neurosurg Psychiatry 2016;87:1005-15.

11 Gaetani L, Blennow K, Calabresi P, et al. Neurofilament light chain as a biomarker in neurological disorders. J Neurol Neurosurg Psychiatry 2019;90:870-81.

12 Novakova L. Neurofilament light for measuring the treatment efficacy in clinical practice: are we there yet? J Neurol Neurosurg Psychiatry 2019;90:1305. 